DOI: https://doi.org/10.24297/jap.v16i1.8273

\title{
The Effect of Suction and Injection on MHD Flow Between Two Porous Concentric Cylinders Filled with Porous Medium
}

\author{
S.E.E. Hamza \\ Physics Department, Faculty of Science, Benha University, Benha, Egypt \\ salah.hamza@fsc.bu.edu.eg
}

\begin{abstract}
This Paper deals wit the effects of suction (injection) on magnetohydrodynamic (MHD) steady flow of a viscous and electrically conducting fluid in an annular porous region between two concentric cylinders. The inner cylinder is rotating with uniform angular velocity and the outer one is fixed. The two cylinders are porous with uniform permeability. It is assumed that the suction rate at the inner cylinder is equal to the injection rate at the outer cylinder. A uniform axial magnetic field was applied perpendicular to the flow direction. The flow resistance presented by the porous medium is governed by the Darcy law. By using similarity transformation, the governing partial differential equations have been transformed to a system of nonlinear ordinary differential equations. The solution of the obtained system in its general form has been obtained. Analytical expression for velocity field is obtained in terms of Bessel function of first and second kind. The effects of various parameters such as susction (injection), magnetic and permeability parameters on the flow are discussed and the obtained results are presented graphically. The obtained figures show that, the velocity distribution increased with the increase of permeability parameter of the the porous medium and with suction process. On the other hand, the velocity distribution decreased with the increase of magnetic parameter and with injection parameter.
\end{abstract}

\section{Keywords}

Concentric cylinders, Navier Stocks equation, Suction, Injection, MHD flow, Bessel function, Porous medium, Darcy law, Permeability parameter, Magnetic parameter.

\section{Academic Discipline And Sub-Disciplines}

Physics, Fluid Mechanics

\section{Subject Classification}

Rheology of Polymers

\section{Type (Method/Approach)}

Theoretical Study.

\section{Introduction}

Flow in porous medium has been extensively investigated due to its numerous applications in geophysics, petroleum industry and chemical engineering etc. Many authors deal with the flow through porous cylinders [1-7]. It is known that even for the Newtonian fluid, if the cylinder surfaces are porous, a uniform suction applied on it can sensibly change the boundary layer structure, reduce the drag and hinder viscous diffusion of vorticity, $[8,9]$. The flows of many other fluid models have been studied in this geometry, but we shall not discuss them here, [10, 11]. Terrill [12] carried out a detailed study of the laminar fow through a porous 
annulus by assuming the swirl to be zero and presented a series solution for small suction or injection. In recent years, the requirements of modern technology have stimulated interest in fluid flow studies, which involve the interaction of several phenomena. One such study is related to the flows of fluid through porous medium due to their applications in many branches in science and technology, viz. in the fields of agricultural engineering to study the underground water resources, in petroleum technology to study the movement of natural gas, oil, water through the oil reservoirs and in chemical engineering for filtration and purification processes. Such problems have also important applications in geo-thermals reservoirs and geo-thermal energy extractions. It is obvious that in order to utilize the geo-thermal energy to maximum, one should have a complete and precise knowledge of the amount of perturbations needed to generate flow in geo-thermal fluids. Abu-hijleh $[13,14]$ analyzed convection heat transfer from a cylinder with porous medium. Hamza et al. [15] have concedred Poiseuille flow between two coaxial porous cylinders with slip on inner cylinder. Sharma et al. [16] investigates the unsteady flow of viscous incompressible fluid through porous medium induced by periodically heated half filled concentric cylindrical annulus placed horizontally. The effect of porous inserts on the natural convection heat transfer in a vertical open-ended annulus has been numerically investigated by Kiwan and Al-Zahrani [17]. Recently, the problem of two-phase unsteady MHD flow between two concentric cylinders of infinite length has been analysed by tha et al. [18] when the outer cylinder is impulsively started. Some recent available literature dealing with the flows in second grade fluid can be found in Tan and Masuoka [19], Fetecau and Fetecau [20], Hayat et al. [21], Erdogan and Imrak [22], Sahoo [23], Ariel [24], Hayat et al. [25, 26, 27]. The effects of porous medium have been investigated by Hayat et al. [28] on the steady flow of a third grade fluid between two stationary porous plates. The governing nonlinear equations are solved by a homotopy analysis method.

The effect of magnetic field on the flow becomes important when the flowing fluid is conducting fluid. An understanding of MHD flows is important to the control of liquid metal pumps, of MHD power generators, and of liquid metal heat exchangers in nuclear fusion reactors. Experiments and numerical simulations have been carried out to reveal the behavior of flow under the influence of a magnetic field. Mahapatra [29] has investigated the problems of unsteady motion of a viscous conducting liquid between two porous nonconducting infinite concentric circular cylinders rotating with various angular velocities for some time in presence of a radial field. Khan et al. [30] found the analytic solution for flow of a MHD Sisko fluid through a porous medium by introducing the Darcy's law using the homotopy analysis method. In the limiting case, the obtained solution reduces to the well known solutions for a Newtonian fluid in non porous and porous media. Hayat et al. [31] investigated the MHD flow of a non-Newtonian fluid filling up the porous space in achannel with compliant walls. They used constitutive equations of a Jeffery fluid. Pantokatoras and Fang [32] investigate the Poiseuille and Couette flow in a fluid saturated Darcy-Brinkman porous medium channel with an electrically conducting fluid under the action of a magnetic and electric field. Exact analytical solutions are derived for fluid velocity. Zhao et al. [33] extend the previous work [32] to the case with a Darcy-BrinkmanForchheimer porous medium. Srivastava et.al. [34] have concedred Poiseuille and Couette flow of an electrically conducting fluid through a porous medium of variable permeability under the transverse magnetic field. They used the Brinkman equation for flow through the porous medium and obtained a numerical solution for velocity and the volumetric flow rate using the Galerkin method.

Magnetic fluids can be considered as liquid mixtures made of magnetic particles chains and small molecules of solvent. At rest, the chains of suspensions are randomly entangled and they do not set up the suspensions structure. When the fluid is in motion, the chains tend to align themselves parallel to the direction of flow. This tendency increases with increasing shear rate, so that the effective viscosity decreases $[35,36]$. The orientation of magnetic particles in solvent under the influence of an external magnetic field is of great importance owing to the possibility of changes in structure and the products formation. Under the effect of magnetic field these particles may rearrange themselves taking the same direction as the magnetic field lines (i.e. oriented parallel to the magnetic lines), a circumstance that lead to an increase in suspension viscosity. Sheikhzadeh et al. [37] studied numerically the flow field and the heat transfer of a non Newtonian fluid in an axisymmetric channel with a permeable wall. Santhosh et al. $[38,39]$ investigate the two fluid model for the flow of a Jeffrey fluid in tubes of small diameters in the presence of a magnetic field. 
Fakour et al. [40] studied the heat transfer process in nanofluid and MHD flow in a channel with permeable walls. Aberkane et al. [41] study numerically the effect of an axial magnetic field imposed on incompressible flow of electrically conductive fluid between two horizontal coaxial cylinders. The effect of heat generation due to viscous dissipation is also taken into account. A finite difference implicit sheme was used in the numerical solution to solve the governing equations of convection flow and mass transfer. Aminfar et al. [42] studied experimentally the effects of using magnetic nanofluid and also applying an external magnetic field on the critical heat flux of sub-cooled flow boiling in vertical annulus. Seth and Singh [43] studied theoretically the effect of Hall current and a uniform transverse magnetic field on unsteady MHD Couette flow of class-II in a rotating system. Verma and Dixit [44] have concerned the MHD laminar steady flow of a viscous incompressible fluid in an annular porous region between two coaxial cylindrical pipes under the uniform transverse magnetic field. Beg et al. [45] presented a mathematical model for the steady, axisymmetric MHD flow of viscous, Newtonian, incompressible, electrically conducting liquid in a high porous regime intercalated between two concentric rotating cylinders in the presence of a radial magnetic field. The flow field of a thirdgrad non-Newtonian fluid in the annulus of rotating concentric cylinders has been investigated by Dizaji et al. [46] in the presence of magnetic field.

In the present problem we have studied the steady and laminar flow of a viscous, incompressible fluid in an annular porous concentric cylinders filled saturated porous medium under the uniform axial magnetic field. Our interest is in understanding the interaction between the viscous fluid and porous medium and the effect of suction or injection at the boundary. Exact solution is obtained and the results are presented for many cases. The Darcy's law is used for flow through a porous medium. The velocity field is obtained and exhibited graphically. The efect of various parameters has been analysed. It has been shown that, the efects of permeability and magnetic parameters have strong effects on flow characteristics.

\section{Formulation of the Problem}

We consider here the steady flow of an electrically conducting viscous incompressible fluid contained between two concentric porous cylinders which is filled with a porous medium. The inner cylinder is rotating with uniform angular velocity $\Omega$ around the system axis and the outer one is fixed. The walls of the cylinders being porous with uniform permeability. We use cylindrical coordinate system $(r, \theta, z)$ with the $z$-axis coincident with the common axis of the cylinders. We assume radius of inner and outer cylinder is $R_{1}$ and $R_{2}$, respectively. $A$ uniform axial magnetic field $\underline{B}$ of strength $B_{0}$ is acting on the axial direction, figure 1 . In the analysis, we assume that the induced magnetic field is negligible.
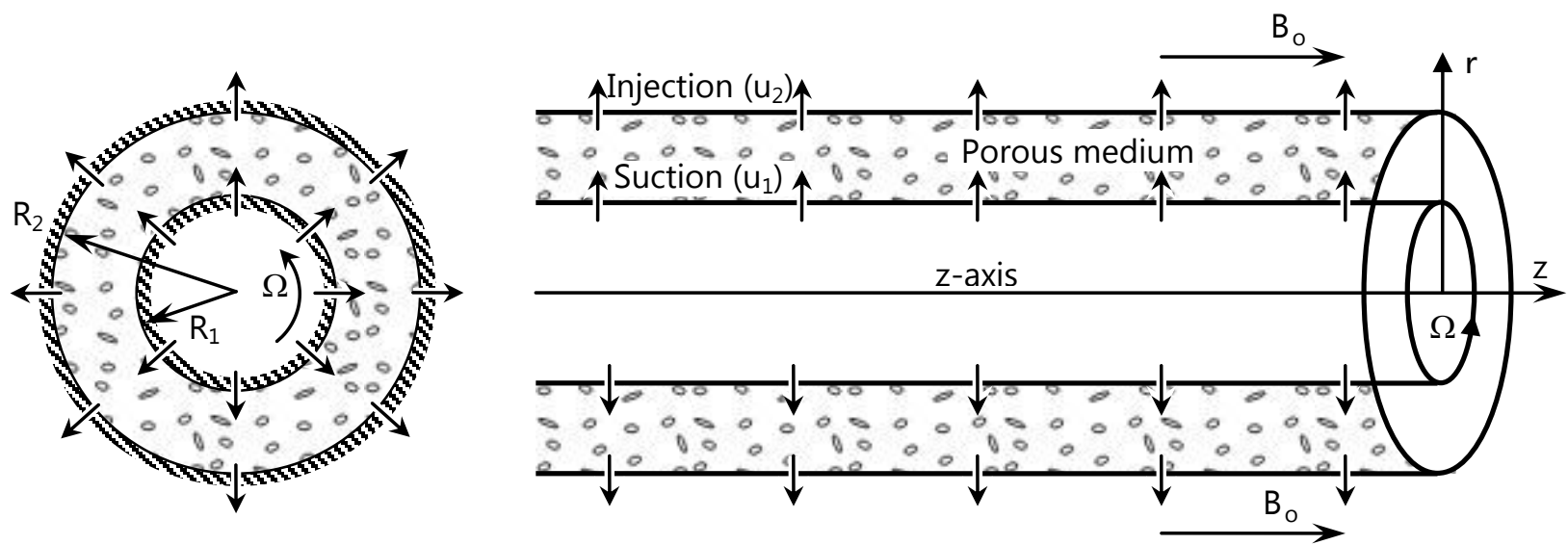

Fig 1: Schematic diagram of the problem and cross section of cylinders.

For steady flow the equations governing the flow are the continuity and momentum equations: 


$$
\begin{aligned}
& \nabla \cdot \underline{\mathrm{V}}=0, \\
& \rho(\underline{\mathrm{V}} \cdot \nabla \underline{\mathrm{V}})=-\nabla \mathrm{p}+\mu \nabla^{2} \underline{\mathrm{v}}+\underline{\mathrm{f}},
\end{aligned}
$$

here $\underline{V}$ is the velocity, $p$ is the pressure, $\mu$ is the coefficient of viscosity and $\underline{f}$ is the body force per unit volume. The presence of a magnetic field and porous medium require that an additional forces be included in the equations of fluid motion aside from the usual pressure and shear forces. The added force takes the form:

$$
\begin{aligned}
& \underline{f}=\underline{J} \times \underline{B}+\underline{R}, \\
& \underline{R}=-\frac{\mu \phi}{k} \underline{V},
\end{aligned}
$$

where $\underline{J}$ is the current density, $\underline{B}$ is the magnetic induction vector, $\underline{\mathfrak{R}}$ is the Darcy resistance for the fluid in the porous medium, $\phi$ and $k$ are the porousity and the permeability of the porous medium. The current density may be expressed by the generalized Ohm's law as:

$$
\underline{J}=\sigma(\underline{E}+\underline{V} \times \underline{B}) ，
$$

in which the terms $\sigma \underline{E}$ and $\sigma(\underline{V} \times \underline{B})$, respectively, represent the conduction and induction currents and $\sigma$ is the electrical conductivity. In the present study, we assume that the magnetic field is in z-direction. Therefore, neglecting the electric field $\underline{E}$ in equation 4 and replacing $\underline{B}$ by the externally applied field

$$
\underline{B}=B_{0} \hat{z} \text {. }
$$

We shall seek an axisymmetric two dimentional solution and thus assume that all variables are independent on the coordinate $\theta$ due to the symmetry about the z-axis.Therefore, In cylindrical coordinates the velocity field, magnetic force and and the Darcy resistance may be written as:

$$
\begin{aligned}
& \underline{\mathrm{V}}=[u(r), u(r)], \\
& \underline{\mathrm{V}} \times \underline{\mathrm{B}}=-\sigma \mathrm{B}_{\mathrm{o}}^{2}[u(r), u(r)], \\
& \underline{\mathfrak{R}}=\left[\mathfrak{R}_{r}, \mathfrak{R}_{\theta}\right]=-\frac{\mu \phi}{\mathrm{k}}[u(r), u(r)] .
\end{aligned}
$$

Therefore, the governing equations 1 and 2 in component form are:

$$
\begin{aligned}
& \frac{d(r u)}{d r}=0, \\
& \rho\left(u \frac{d u}{d r}-\frac{v^{2}}{r}\right)=-\nabla p+\mu\left(\frac{d^{2} u}{d r^{2}}+\frac{1}{r} \frac{d u}{d r}-\frac{u}{r^{2}}\right)-\sigma B_{o}^{2} u-\frac{\mu \phi}{k} u, \\
& \rho\left(u \frac{d v}{d r}+\frac{u v}{r}\right)=\mu\left(\frac{d^{2} v}{d r^{2}}+\frac{1}{r} \frac{d v}{d r}-\frac{v}{r^{2}}\right)-\sigma B_{o}^{2} v-\frac{\mu \phi}{k} v .
\end{aligned}
$$

We assume that the inner cylinder is rotating with angular velocity $\Omega=\Omega \hat{z}$ while the outer one is kept stationary. The angular velocity at the inner cylinder $\underline{\underline{v}}\left(R_{1}\right)=\Omega \hat{z} \wedge R_{1} \hat{r}=R_{1} \Omega \hat{\theta}$ while at the outer cylinder 
vanishes $v\left(R_{2}\right)=0$. The cylinders have porous walls and a constant radial velocity $u_{1}$ is applied to the inner one, denoting a uniform suction $\left(u_{1}<0\right)$ or injection $\left(u_{1}>0\right)$. A suction at the inner wall corresponds to an injection at the outer one and vice versa. Therefore, the boundary conditions can be formulated as:

$$
u\left(R_{1}\right)=u_{1}, \quad v\left(R_{1}\right)=R_{1} \Omega, \quad v\left(R_{2}\right)=0 .
$$

The solution of the continuity equation, 9 , gives

$$
\mathrm{u}=\frac{\mathrm{R}_{1} \mathrm{u}_{1}}{\mathrm{r}}
$$

the last equation satisfy the boundary conditions, $u\left(R_{1}\right)=u_{1}$, which represents the suction value at the inner cylinder and gives the radial velocity component at $R_{2}$ as $u\left(R_{2}\right)=\frac{R_{1} u_{1}}{R_{2}}$ which represents the injection value at the outer cylinder.

The following dimensionless quantities are used:

$$
r^{*}=\frac{r}{R_{1}}, \quad u^{*}=\frac{u}{u_{1}}, \quad v^{*}=\frac{v}{R_{1} \Omega}, \quad p^{*}=\frac{p}{\rho\left(R_{1} \Omega\right)^{2}},
$$

after straightforward computations it follows that the dimensionless radial velocity $u$ is given by (after dropping the dimensionless mark "*" for simplicity):

$$
u=\frac{1}{r}
$$

and the dimensionless azimuthal velocity $v$ satisfies the boundary value problem:

$$
\frac{d^{2} v}{d r^{2}}+\frac{(1-S)}{r} \frac{d v}{d r}-\frac{(1+S)}{r^{2}} v-\beta^{2} v=0
$$

with the boundary conditions

$$
v(1)=1, \quad v(\gamma)=0,
$$

where

$$
S=\frac{\rho R_{1} u_{1}}{\mu}, \quad \beta^{2}=M+\frac{1}{K}, \quad M=\frac{\rho B_{o}^{2} R_{1}^{2}}{\mu}, \quad \frac{1}{K}=\frac{\phi R_{1}^{2}}{k}, \quad \gamma=\frac{R_{2}}{R_{1}},
$$

and $\mathrm{S}$ is the suction (injection) parameter, $\mathrm{M}$ is the magnetic parameter and $\mathrm{K}$ is permeability parameter. The values of $\mathrm{M}$ and $\mathrm{K}$ are an index to the relative importance of magnetic forces and permeability of the porous medium respectively. When $M=0$, magnetic forces are absent; when $M$ increases, the magnetic force becomes increasingly important. The value $\mathrm{K}=0$ is for blocked medium (solid); and when $\mathrm{K} \rightarrow \infty$ (or $1 / K \rightarrow 0$ ) the annular region beween the two cylinders becomes a hollow cylinder. 


\section{Solution of the Problem}

Equation 16 is to be solved subject to the boundary conditions in equation 17. Also equation 16 can be reduced and an exact solution can be found. The classical Couette flow (Newtonian fluid without suction, mamnetic field and porous medium) is obtained as a special case.

\section{Special Cases}

\section{(a) Couette flow with suction (injection) only}

In this case $\beta=0$ (there is no magnetic field $M=0$ and hollow region between the two cylinders $K \rightarrow \infty$ ). Therefore, equation 16 takes the form:

$$
\frac{d^{2} v}{d r^{2}}+\frac{(1-S)}{r} \frac{d v}{d r}-\frac{(1+S)}{r^{2}} v=0
$$

the solution of the last equation is:

$$
v(r)=\frac{r^{S+2}-\gamma^{S+2}}{r\left(1-\gamma^{S+2}\right)} \text {. }
$$

\section{(b) Couette flow with magnetic field and porous medium}

For no suction (injection) an analytical solution of the system of equations 16 and 17 can be found. In this case $\mathrm{S}=0$ and the governing equation is:

$$
\frac{d^{2} v}{d r^{2}}+\frac{1}{r} \frac{d v}{d r}-\frac{1}{r^{2}} v-\beta^{2} v=0
$$

the solution of the last equation is:

$$
v(r)=A_{1} J_{1}(i \beta r)+A_{2} Y_{1}(-i \beta r),
$$

where $J_{1}$ and $Y_{1}$ are the Bessel functions of first and second kind, respectively. Using the boundary conditions $17, A_{1}$ and $A_{2}$ are obtained as follows

$$
\begin{aligned}
& A_{1}=\frac{-Y_{1}(-i \beta \gamma)}{J_{1}(i \beta \gamma) Y_{1}(-i \beta)-J_{1}(i \beta) Y_{1}(-i \beta \gamma)}, \\
& A_{2}=\frac{J_{1}(i \beta \gamma)}{J_{1}(i \beta \gamma) Y_{1}(-i \beta)-J_{1}(i \beta) Y_{1}(-i \beta \gamma)} .
\end{aligned}
$$

\section{(c) Couette flow with suction (injection), magnetic field and porous medium}

In this case the general solution of the governing equations 16 and 17 is:

$$
v(r)=r^{\frac{S}{2}}\left[C_{1} J_{\frac{S+2}{2}}(-i \beta r)+C_{2} Y_{\frac{S+2}{2}}(-i \beta r)\right]
$$


where $\mathrm{J}_{\frac{S+2}{2}}$ and $\mathrm{Y}_{\frac{S+2}{2}}$ are the Bessel functions of order $\frac{S+2}{2}$ of first and second kind, respectively. $C_{1}$ and $C_{2}$ are constants of integration. Introducing the boundary conditions 17, to equation 25 we have

$$
\begin{aligned}
& C_{1}=\frac{-Y_{\frac{S+2}{2}}(-i \beta \gamma)}{J_{\frac{S+2}{2}}(i \beta \gamma) Y_{\frac{S+2}{2}}(-i \beta)-J_{\frac{S+2}{2}}(i \beta) Y_{\frac{S+2}{2}}(-i \beta \gamma)}, \\
& C_{2}=\frac{J_{\frac{S+2}{2}}(i \beta \gamma)}{J_{\frac{S+2}{2}}(i \beta \gamma) Y_{\frac{S+2}{2}}(-i \beta)-J_{\frac{S+2}{2}}(i \beta) Y_{\frac{S+2}{2}}(-i \beta \gamma)},
\end{aligned}
$$

\section{Results and Discussion}

We noticed a strong correlation among the many parameters upon which the flow depends. Among a variety of numerical experiments we report here only the most significant: we always fixed $R_{2}=2 R_{1}$ $(\gamma=2), S=0, \pm 0.1, \pm 0.3, \pm 0.6, \pm 1$ (negative values for suction and positive values for injection), $\mathrm{M}=0.1,0.2,0.4,0.8,1.2$ and $\mathrm{K}=0.03,0.1,0.2,0.5,0.9$. A brief description of the efforts due to changes of each parameter is given below. In order to understand the physical situation of the problem and the effect of suction (injection), magnetic and porousity parameters, we have found the variation of velocity field for $1 \leq r \leq \gamma$ in all special cases and for values of the included physical parameters.

The variation of velocity profile with suction and injection parameter $\mathrm{S}$ for fixed value $\mathrm{M}=0$ and $\frac{1}{\mathrm{~K}} \rightarrow 0$ (or $\beta=0$ ) is shown in figures 2 and 3 for $\gamma=2$. We observe that velocity profile decreases with increment in suction and increases with increasing injection in annular region of concentric cylinders. This is because increase in $\mathrm{S}$ means decrease in the permeability of the porous medium. For $\mathrm{S}<0$ (suction) a strong departure of the solution is obtained from that for the case $S=0$. The velocity profile becomes steeper and steeper at the inner wall as $\mathrm{S}$ grows. The fluid particles are pushed towards the inner wall and the velocity $v$ can overshoot values at the boundaries, figure 2. A similar effect is evidenced for $\mathrm{S}>0$ (injection). The fluid particles are deriven to the outer wall, but it turns out that there is no solution if $S$ exceeds some critical value, figure 3.

Figures 4-6 give the velocity profiles in the presence of magnetic field for various values of suction (injection) parameter $S$. The influence of magnetic parameter $M$ on the velocity profile is presented in figure 4 for $S=0$ and $\mathrm{K}=0.5$. From these figures, it is noted that an increase in magnetic parameter $M$ reduces the velocity profile monotonically due to the effect of the magnetic force against the direction of the flow. It is clear that, the velocity profile without magnetic field, $M=0$, is quasi linear. An increase in magnetic parameter, which causes a reduction of the velocity in the annular space because the centrifugal force, is counter productive and the Lorentz electromagnetic foces acts as a flow damper. The effect of magnetic parameter $M$ on the flow velocity for $\mathrm{S}=-0.6$ and $\mathrm{K}=0.5$ is shown in figure 5 . It is clear that, the velocity decreases with increase in magnetic parameter. The same behaviour is shown in figure 6 for $S=0.6$ and $K=0.5$. This is because of the fact that increase in magnetic field increases the Lorentz force in opposite to the direction of flow. So we can use magnetic field to control the fluid velocity.

The enfluence of permeability parameter $K$ on the flow velocity for fixed $S=0$ and $M=0.4$ is depicated in figure 7. From the figure we can observe that the velocity is showing increasing behavior with the increasing the value of $K$. The same behavour is observed in the presence of suction, $S=-0.6$, figure 8 , and injection $S=0.6$, figure 9 , for fixed $M=0.4$. This is because permeability $K$ increases as $\beta$ decreases. 


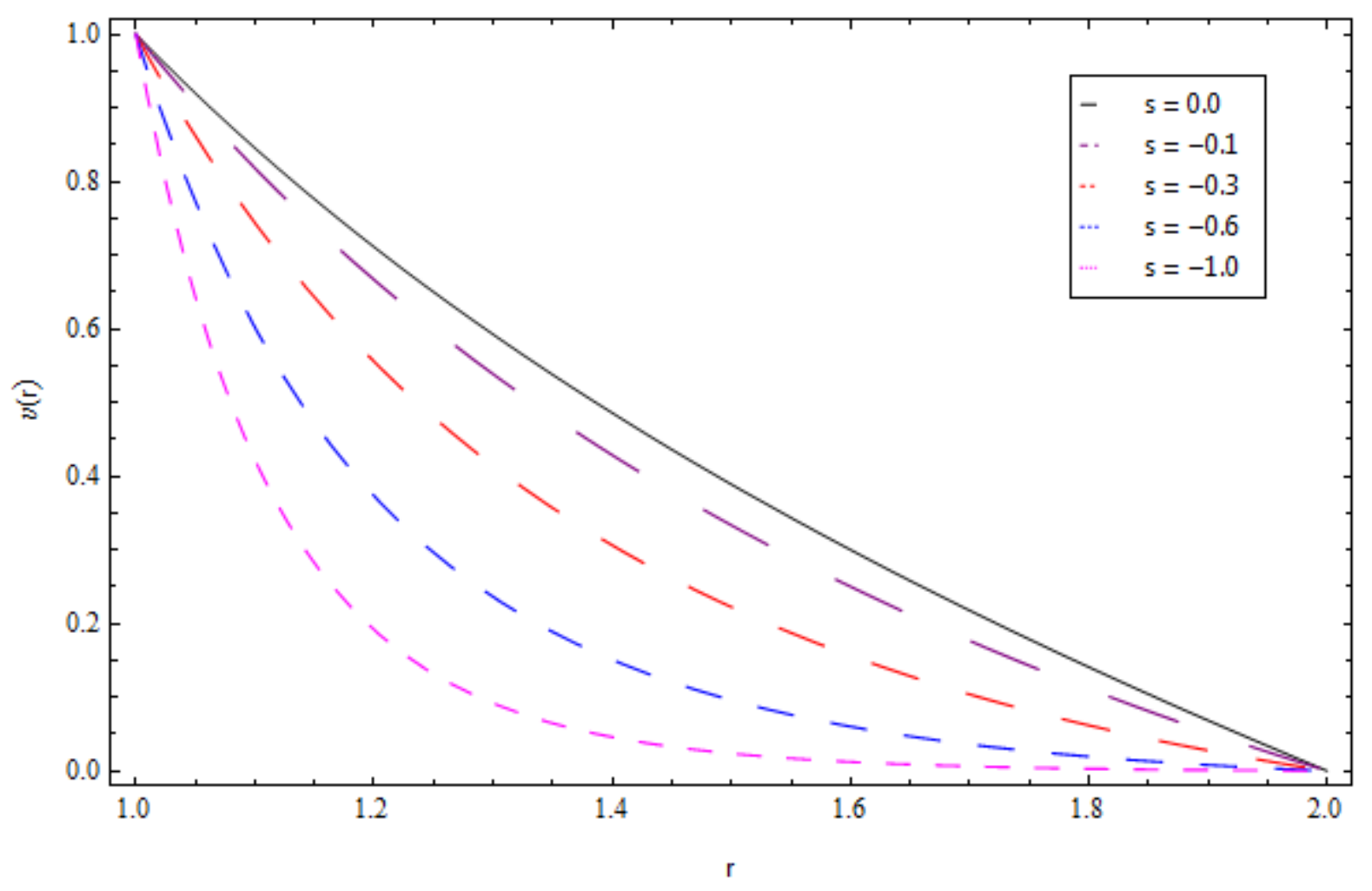

Fig 2: Effect of suction parameter on velocity profiles of the fluid in the annulus for $\gamma=2$ and $\beta=0$.

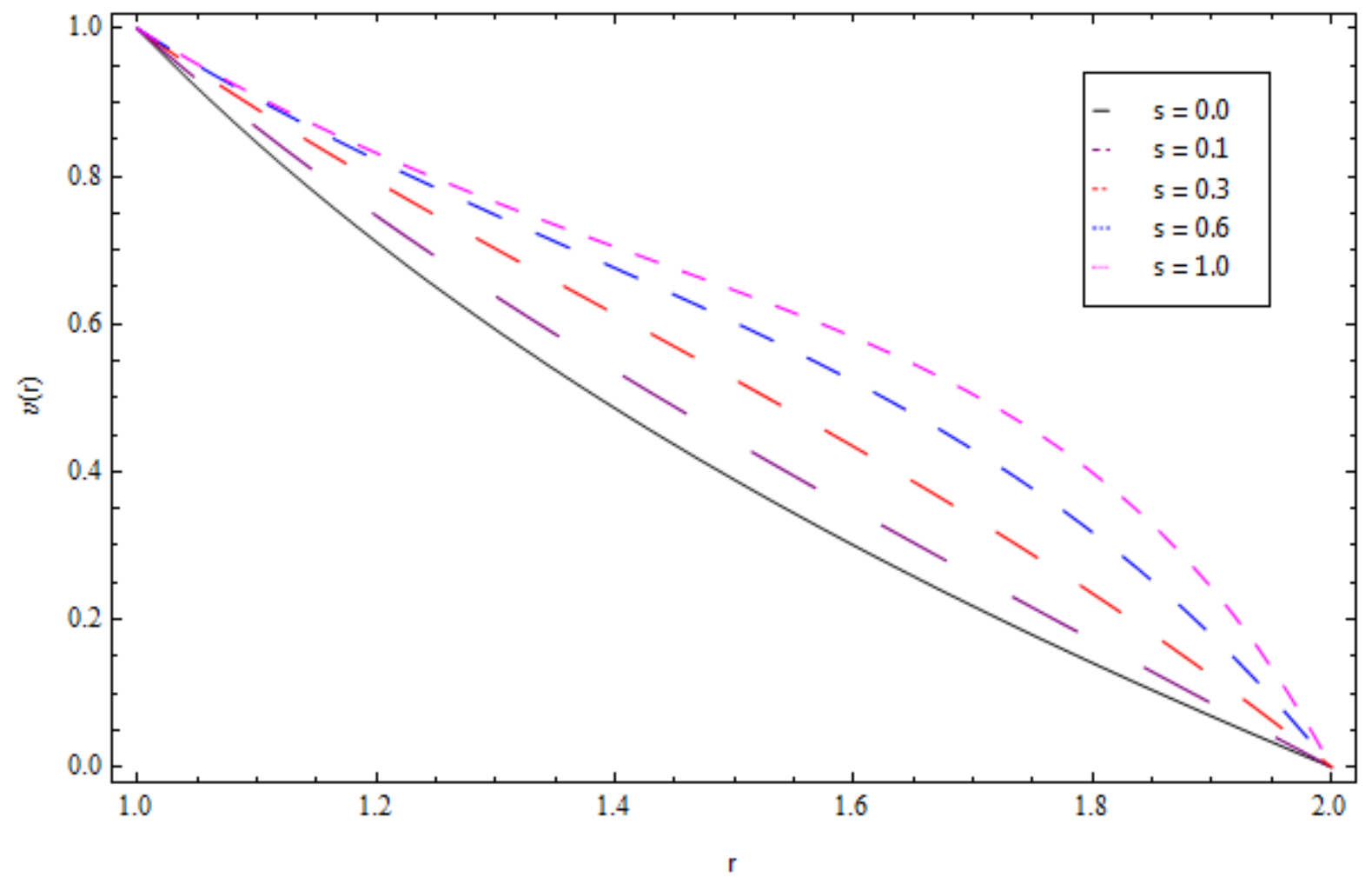

Fig 3: Effect of injection parameter on velocity profiles of the fluid in the annulus for $\gamma=2$ and $\beta=0$. 


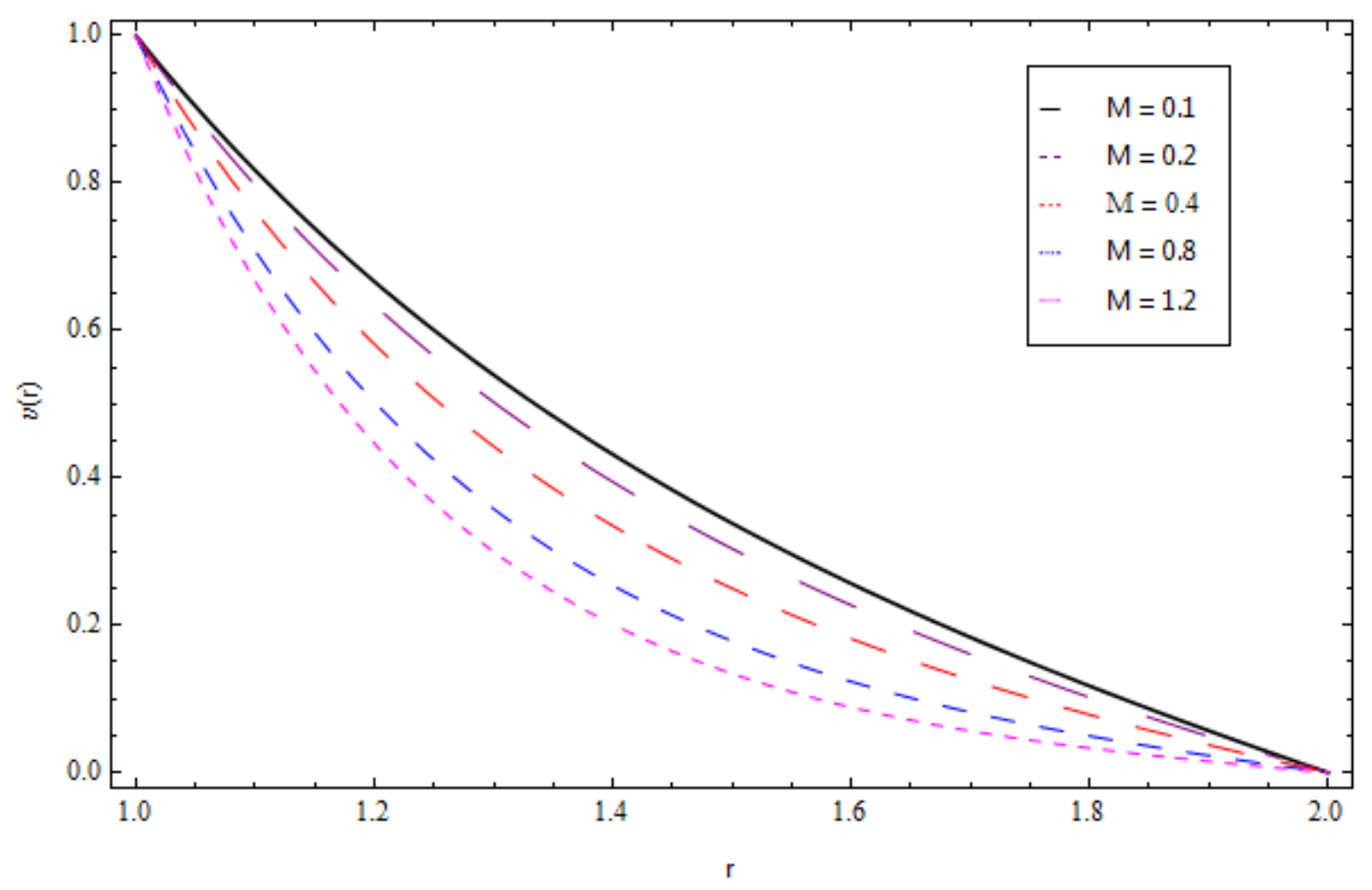

Fig 4: Effect of magnetic parameter on velocity profiles for $S=0$ and $K=0.5$.

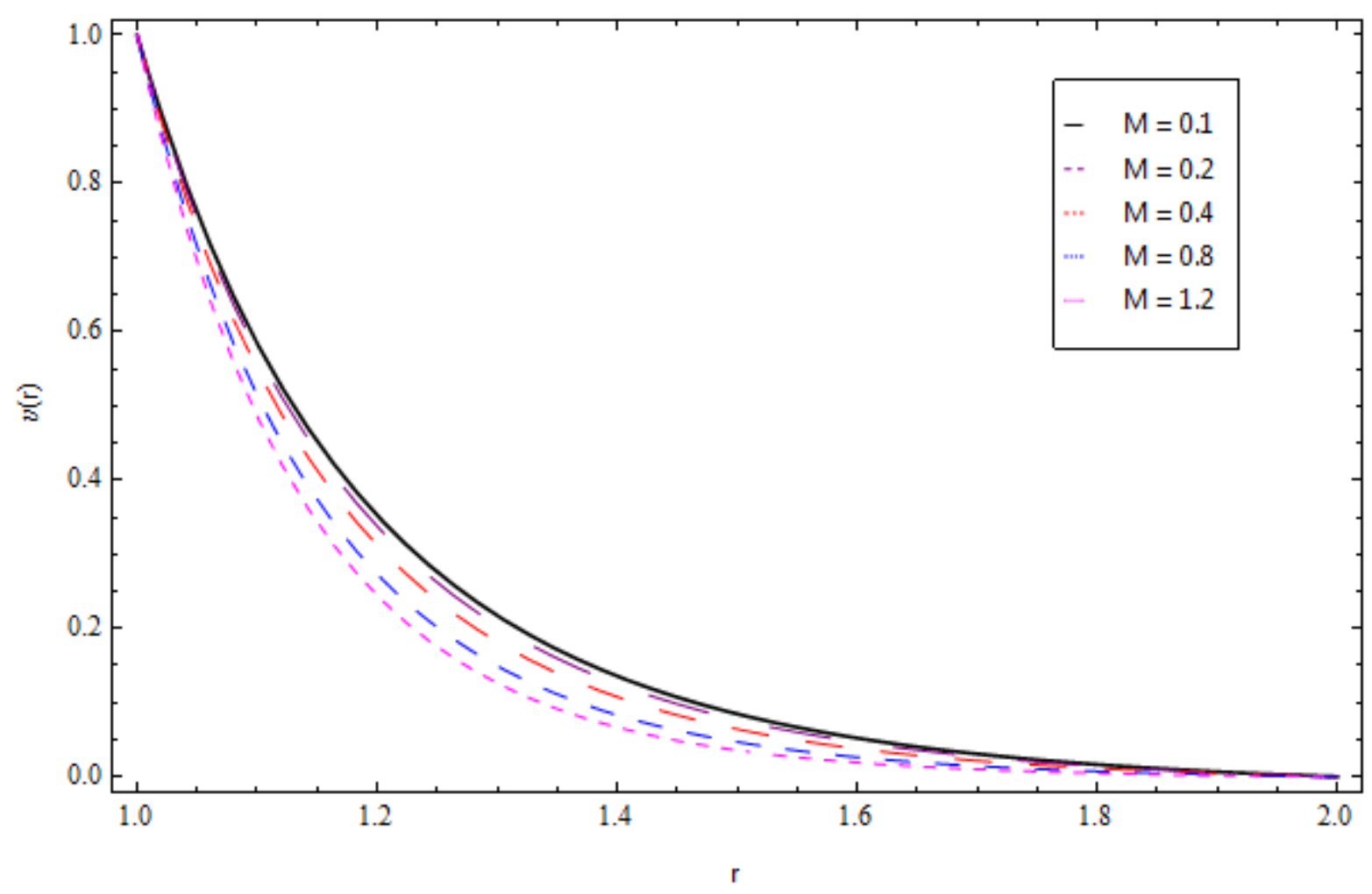

Fig 5: Effect of magnetic parameter on velocity profiles for $S=-0.6$ and $K=0.5$. 


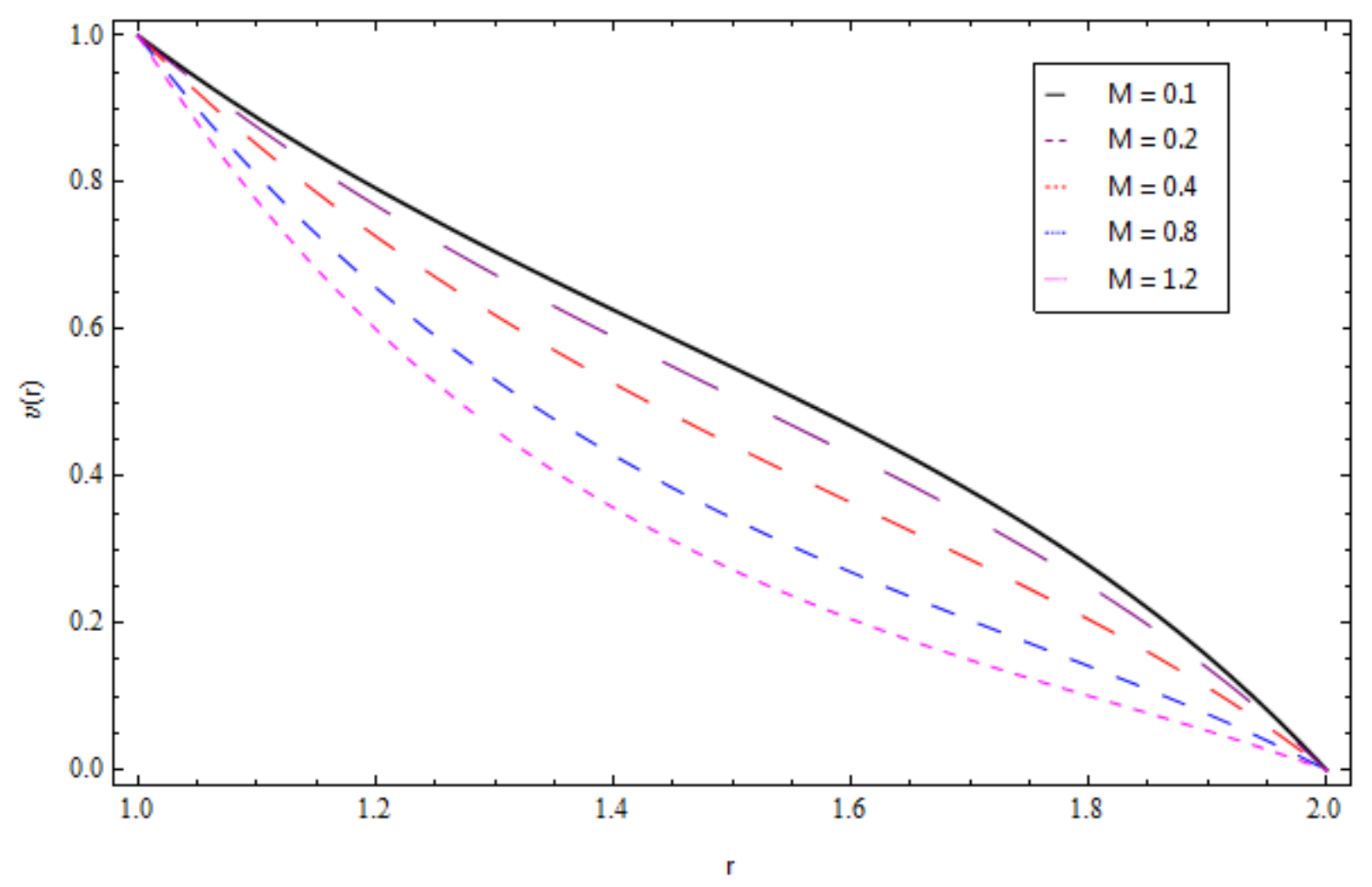

Fig 6: Effect of magnetic parameter on velocity profiles of the fluid in the annulus for $S=0.6$ and $\mathrm{K}=0.5$.

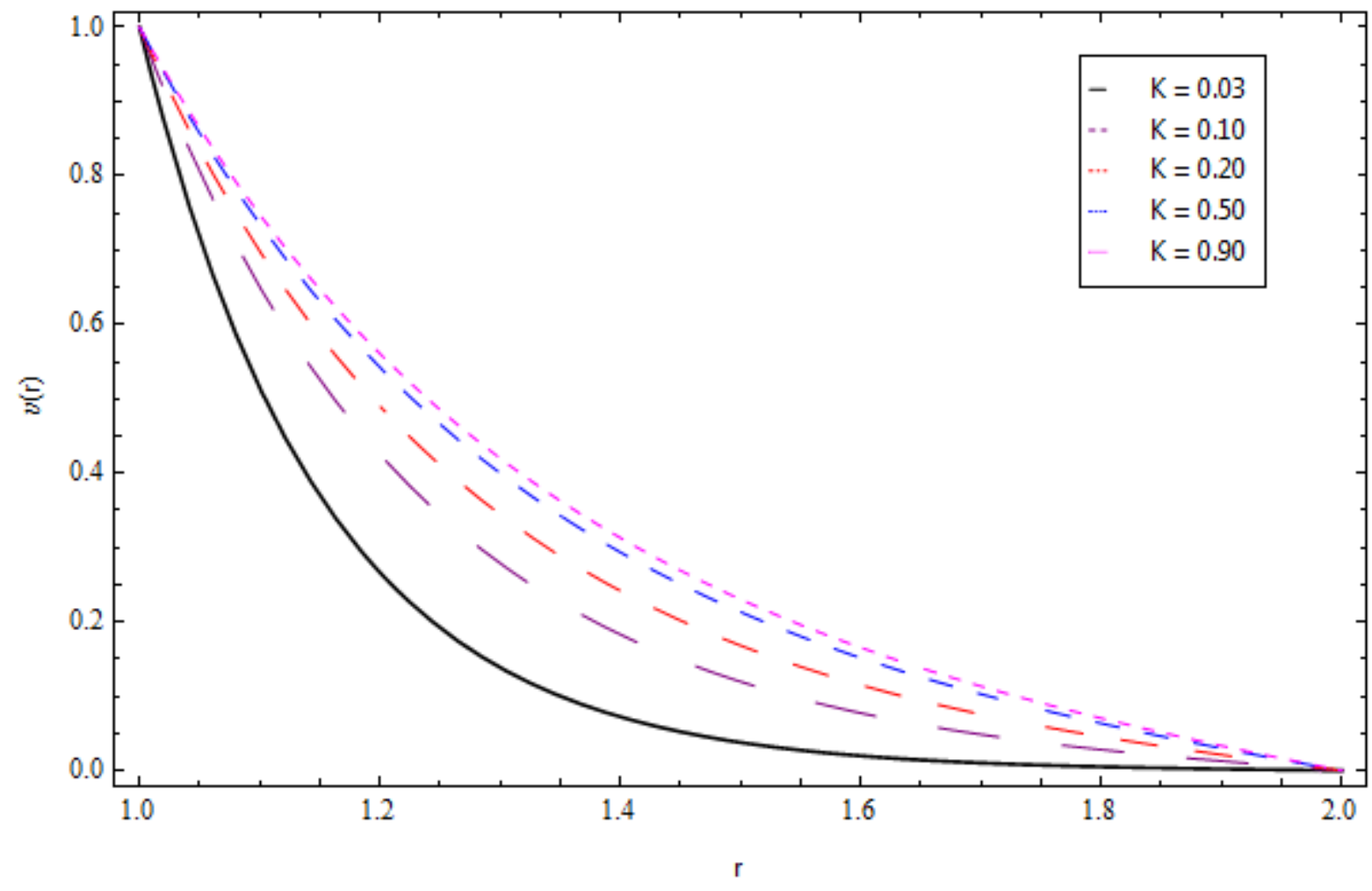

Fig 7: Effect of permeability on velocity profiles of the fluid in the annulus for $S=0$ and $M=0.4$. 


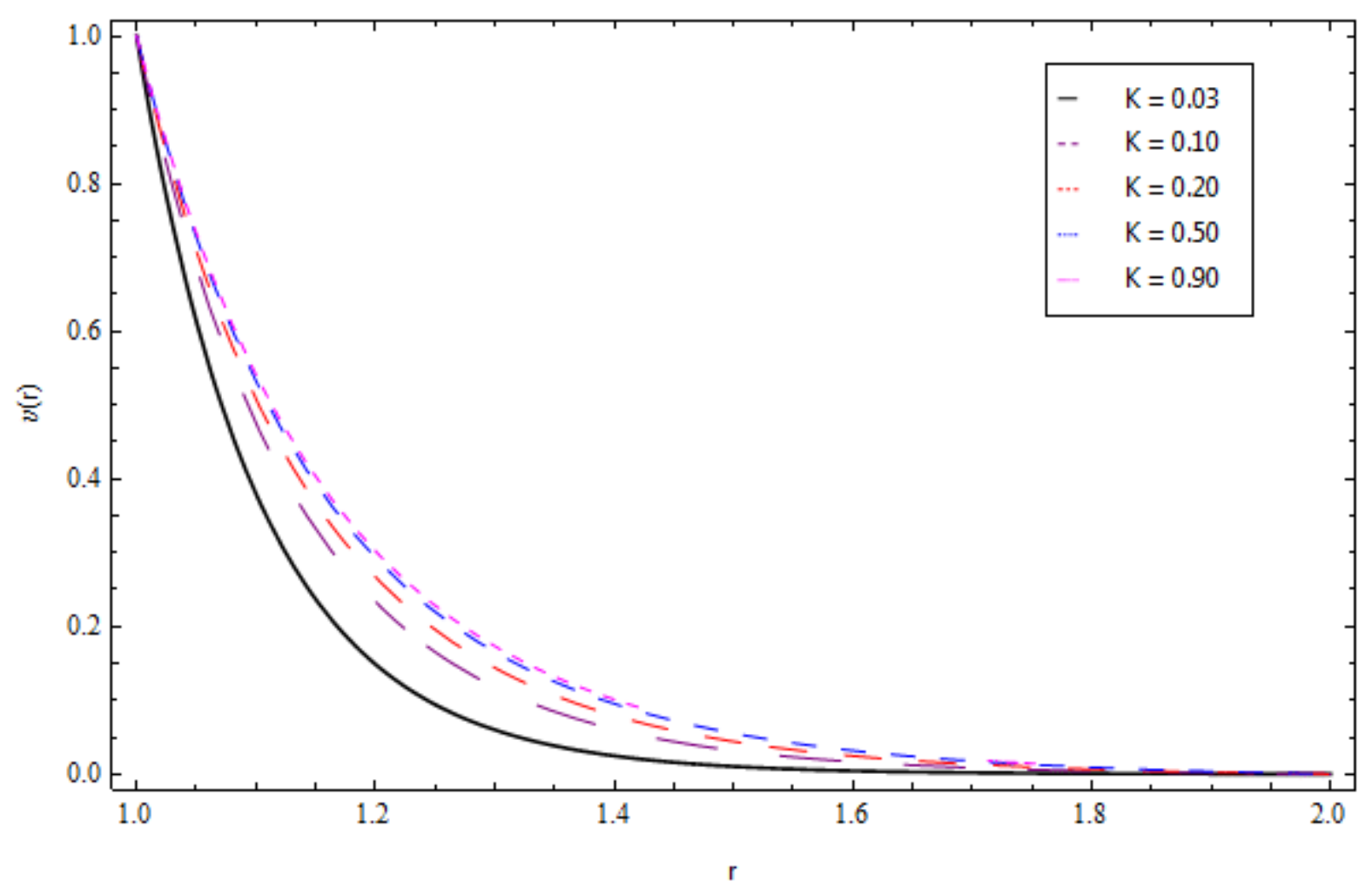

Fig 8: Effect of permeability on velocity profiles for $S=-0.6$ and $M=0.4$.

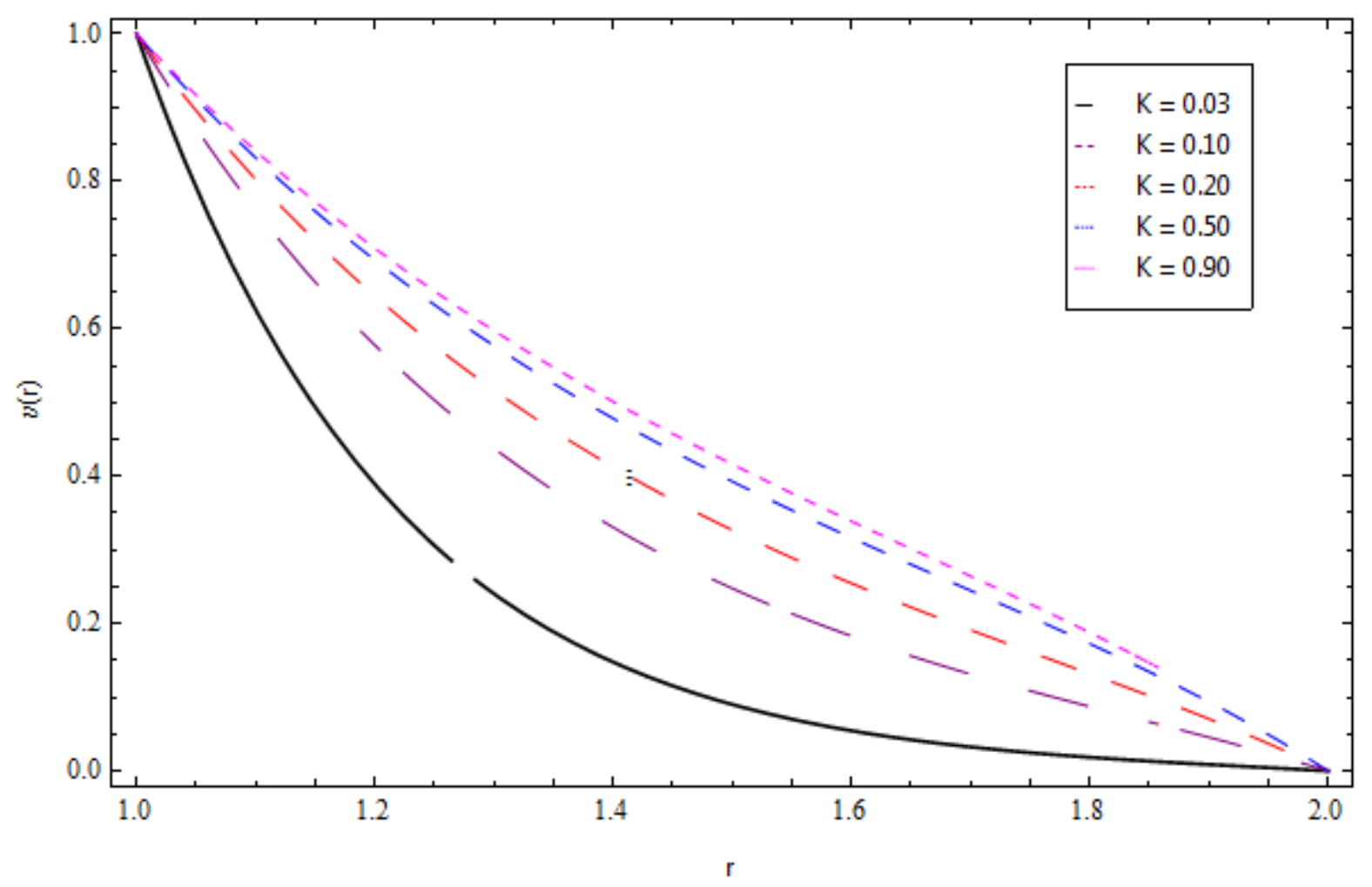

Fig 9: Effect of permeability on velocity profiles for $S=0.6$ and $M=0.4$. 


\section{Conclusions}

The main objective of the theoretical solution is to examine in detail the effect of suction and injection on MHD flow of viscous and electrically conducting fluid in an annular porous region between two concentric cylinders. The walls are porous and a suction or injection is applied at one of them. The governing equation are solved in exact manner considering the Lorentz force to account the resistance offered by the magnetic field and Darcy's law to account the flow resistance presented by the porous medium. The obtained velocity profiles possess modified Bessel functions of first and second kind. The results presented show how the solution varies along the flow and exhibits a strong dependence on the suction parameter. The effects of various parameters on the flow characteristics such as suction (injection), magnetic and permeability parameters are studied and obtained results are exhibited graphically. In limiting case when magnetic field and permeability is zero the obtained results reduces to the classical results of Couette flow in an annular cylinder. Therefore, we conclude the following remarks:

- An increase in suction (injection) parameter $\mathrm{S}$ decrease (increase) the velocity profile monotonically.

- An increase in magnetic parameter $M$ reduces the velocity profile monotonically due to the effect of magnetic force against the flow direction. This is because of the fact that increase in magnetic field increases the Lorentz force in opposite to the direction of flow. So we can use magnetic field to control the fluid velocity.

- An increase in the parameter $K$ yields an effect opposite to that of the magnetic field.

\section{References}

[1] Pantokratoras, A. and Fang, T. Flow of a weakly conducting fluid in a channel filled with a porous medium. Transport in Porous Media, 83 ( 2010) 667-676.

[2] Zhao, B. Q., Pantokratoras, A., Fang, T. G. and Liao, S.J. Flow of a weakly conducting fluid in a channel filled with a Darcy - Brinkman - Forchheimer porous medium. Transport in Porous Media, 85 (2010) 131-142.

[3] Verma, V. K. and Datta, S. Flow in a channel filled by heterogeneous porous media with a linear permeability variation. Special Topics and Reviews in Porous Media An International Journal, 3 (3)(2012a) 201-208.

[4] Verma, V. K. and Datta, S. Flow in an annular channel filled with a porous medium of variable permeability. Journal of Porous Media, 15(10) (2012b) 891-899.

[5] Verma, V. K. Analytical solution of magnetohydrodynamic flow with varying viscosity in an annular channel. Adv. Theor. Appl. Math., 9 (2014) 105-122.

[6] Verma, V. K. and Singh, S. Flow between coaxial rotating cylinders filled by porous medium of variable permeability. Special Topics and Reviews in Porous Media An International Journal, 5(4)(2014) 355-359.

[7] Verma, V. K. and Singh, S. Magnetohydrodynamic flow in a circular channel filled with aporous medium. Journal of Porous Media, 18(9) (2015) 923-928.

[8] Fukumoto, Y. General unsteady circulatory flow outside a porous circular cylinder with suction or injection. J. Phys. Soc. Jpn., 59 (1990) 918-926.

[9] Daskalakis, J. E. Mixed free and forced convection in the incompressible boundary layer along a rotating vertical cylinder with fluid injection. Int. J. Energy Res., 17 (1993) 689-695. 
[10] Casarella, M. J. and Laura, P. A. Drag on an oscillating rod with longitudinal and torsional motion. J. Hydronaut., 3 (1969) 180-183.

[11] Rajagopal, K. R. and Kaloni, P. N. Some remarks on boundary conditions for flows of fluids of the differential type. Continuum Mechanics and Its Applications, Hemisphere, Washington, DC, 1989.

[12] Terrill, R. M. Flow through a porous annulus. Appl. Sci. Res. 17 (1967) 204-222.

[13] Abu-hijleh, B. Natural convection heat transfer from a cylinder with high conductivity permeable fins. ASME J. Heat Transfer, 125 (2002) 282-288.

[14] Abu-hijleh, B. Natural convection heat transfer from a cylinder covered with an orthotropic porous layer. Numer. Heat Transfer, 40 (2001) 767-782.

[15] Hamza, E. A., Rajvanshi S. C. and Sacheti, N. C. Pulsatile flow between two coaxial prous cylinders with slip on inner cylinder. Matematicas, XV (1) (2007) 51-66.

[16] Sharma, B. K., Sharma, P. K. and Chaudhary, R. C. Unsteady flow through porous medium induced by periodically rotating half-filled horizontal concentric cylindrical annulus with heat transfer. International Journal of Physical Sciences, 7(10) (2012) 1530-1539.

[17] Kiwan, S and Al-Zahrani, M. S. Effect of porous inserts on natural convection heat transfer between two concentric vertical cylinders. Numer. Heat Transfer Part A, 53 (2008) 870-889.

[18] Jha B. K. and Apere C. A. Unsteady MHD two-phase Couette flow of fluid-particle suspension in an annulus. AIP Advances, 1 (2011) 042121-042136.

[19] Tan, W. C. and Masuoka, T. Stokes first problem for a second grad fluid in a porous half space with heated boundary. Int. J. Non-Linear Mech., 40 (2005) 515-522.

[20] Fetecau, C. and Fetecau, C. Starting solutions for the motion of a second grade fluid due to longitudinal and torsional oscillations of a circular cylinder. Int. J. Eng. Sci. 44 (2006) 788-796.

[21] Hayat, T., Khan, M. and Ayub, M. Some analytical solutions for second grade fluid flows for cylindrical geometries. Math. Comp. Model., 43 (2006) 16-29.

[22] Erdogan, M. E. and Imrak, C. E. On the comparison of two different solutions in the form of series of the governing equation of an unsteady flow of a second grade fluid. Int. J. Non-Linear Mech. 40 (2005) 545550.

[23] Sahoo, B. Effects of slip viscous dissipation and Joule heating on the MHD flow and heat transfer of a second grade fluid past a radially stretching sheet. Appl. Math. Mech., 31 (2010) 159-173.

[24] Ariel, P. D. Flow of a third grade fluid through a porous flat channel. Int. J. Eng. Sci. 41 (2003) 1267-1285.

[25] Hayat, T., Naz, R. and Sajid, M. On the homotopy solution for Poiseuille flow of a fourth grade fluid. Commun Nonlinear Sci. Numer. Simul., 15 (2010) 581-589.

[26] Hayat, T., Asghar, S., Khalique, C. M. and Ellahi, R. Influence of partial slip on flows of a second grade fluid in a porous medium. J. Porous Media, 10 (2007) 797-805.

[27] Hayat, T., Abbas, Z. and Asghar, S. Effects of Hall current and heat transfer on rotating flow of a second grade fluid through a porous medium. Commun. Nonlinear Sci. Numer. Simul., 13 (2008) 2177-2192. 
[28] Hayat, T., Naz, R. and Abbasbandy, S. Poiseuille flow of a third grade fluid in a porous medium. Transport in Porous Media, 87 (2011) 355-366.

[29] Mahapatra J. R. A note on the unsteady motion of a viscous conducting liquid between two porous concentric circular cylinders acted on by a radial magnetic field. Appl. Sci. Res. 27 (1973), 274-282.

[30] Khan, M., Abbas, Z. and Hayat, T. Analytic solution for flow of Sisko fluid through a porous medium. Transport in Porous Media, 71 ( 2008) 23-37.

[31] Hayat, T., Javed, M. and Ali, N. MHD peristaltic transport of a Jeffery fluid in a channel with compliant walls and porous space. Trans Porous Med, 74(2008) 259-274.

[32] Pantokatoras, A. and Fang, T. Flow of weakly conducting fluid in a channel filled with a porous medium. Transport in Porous Media, 83 (2010) 667-676.

[33] Zhao, B. Q., Pantokratoras, A., Fang, T. G. and Liao, S. J. Flow of a weakly conducting fluid in a channel filled with a Darcy-Brinkman-Forchheimer porous medium. Transport in Porous Media, 85 (2010) 131-142.

[34] Srivastava, B. G. and Satya Deo. Effect of magnetic field on the viscous fluid fow in achannel filled with porous medium of variable permeability. A pplied Mathematics and Computation, 219 (2013) 8959-8964.

[35] Ghasemi, E., Mirhabibi, A. and Edrissi, M. Synthesis and rheological properties of an iron oxide ferrofluid. J Magn Magn Mater, 320 (2008) 2635-2639.

[36] Hong, R. Y., Ren, Z. Q., Han, Y. P., Zheng, Y. and Ding, J. Rheological properties of water-based $\mathrm{Fe}_{3} \mathrm{O}_{4}$ ferrofluids. Chem. Eng. Scie., 62 (2007) 5912-5924.

[37] Sheikhzadeh, G. A., Mollamahdi, M. and Abbaszadeh, M. Analytical study of flow field and heat transfer of a non-Newtonian fluid in an axisymmetric channel with a permeable wall. Journal of Computational and Applied Research in Mechanical Engineering, 7(2) (2018) 161-173

[38] Santhosh, N. and Radhakrishnamacharya, G. Jeffrey fluid flow through a narrow tubes in the presence of a magnetic field. Procedia Engineering, 127 (2015) 185-192.

[39] Santhosh, N., Radhakrishnamacharya, G. and Chamkha, A. J. Flow of a Jeffrey fluid through a porous medium in narrow tubes, J. Por. Media., 18(1) (2015) 71-78.

[40] Fakour, M., Ganji, D. D., Khalili, A. and Bakhshi, A. Heat transfer in nanofluid MHD flow in a channel with permeable walls. Heat Transfer Research, 48(3) (2017) 221-228.

[41] Aberkane, S., Ihdene, M., Moderes, M. and Ghezal, A. Effect of an axial magnetic field on the heat and mass transfer in rotating annulus. Int. J. Phys. Sci. 9 (16) (2014) 368-379.

[42] Aminfar, H., Mohammadpourfard, M. and Maroofiazar, R. Experimental study on the effect of magnetic field on critical heat flux of ferrofluid flow boiling in a vertical annulus. Experimental Thermal and fluid Science 58 (2014) 156-169.

[43] Seth, G. S. and Singh, J. K. Effects of Hall current of unsteady MHD Couette flow of class-II in a rotating system. J. Appl. Fluid Mech. 6(4) (2013) 473-484.

[44] Verma, V. K. and Dixit, P. K. MHD Couette flow in annular porous region between two coaxial cylinders. International Journal of Pure and Applied Mathematics, 114 (3) (2017) 571-582. 
[45] Beg, O. A., Makinde, O. D., Zueco, J. and Ghosh, S. K. Hydromagnetic viscous flow in a rotating annular high porosity medium with nonlinear forchheimer drag effects: numerical study. World Journal of Modelling and Simulation, 8 (2) (2012) 83-95.

[46] Dizaji, A. F., Salimpour, M. R. and Jam, F. Flow field of a third-grade non-Newtonian fluid in the annulus of rotating concentric cylinders in the presence of magnetic field. J. Math. Anal. Appl., 337 (2008) 632-645.

\section{Author' biography with Photo}

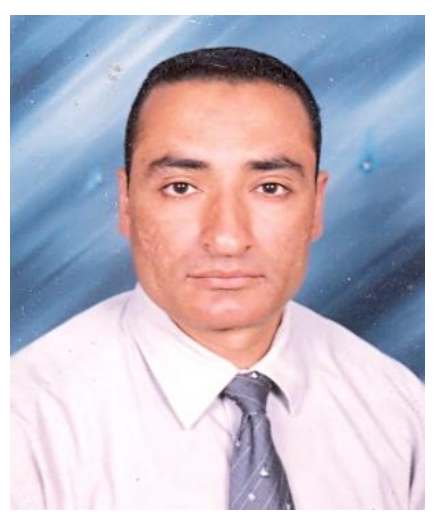

Ass. Prof. S.E.E. Hamza born in Hasanea, Touk, Kalubea, Egypt on $6^{\text {th }}$ January 1968. Since 1991 working at Physics Deparment, Faculty of Science, Benha, Egypt. Obtained his Ph. D. degree in theoretical physics in 2003 from Benha University under the able guidance of Prof. M. Zidan, Prof. A. Abu-El Hassan and Prof. N.M. El Nagar. He is working as Assistant Professor, Faculty of Science, Benha, Egypt in Physics department. Two students, one obtained M.Sc. degree and the other obtained Ph.D. degree under his Guidence. He is guiding one student for M. Sc. The topics of interest are Fluid Dynamics Nonlinear differential equations, BioPhysics. 